\title{
On the Low Energy Decrease in Galactic Cosmic Ray Secondary/Primary Ratios
}

\author{
A.J. Davis ${ }^{1}$, R.A. Mewaldt ${ }^{1}$, W.R. Binns ${ }^{3}$, E.R. Christian ${ }^{2}$, A.C. Cummings ${ }^{1}$, J.S. George ${ }^{1}$, \\ P.L. Hink ${ }^{3}$, R.A. Leske ${ }^{1}$, T.T. von Rosenvinge ${ }^{2}$, M.E. Wiedenbeck ${ }^{4}$, N.E. Yanasak ${ }^{4}$ \\ ${ }^{1}$ California Institute of Technology, Pasadena, CA 91125 \\ ${ }^{2}$ Goddard Space Flight Center, Green Belt, MD 20771 \\ ${ }^{3}$ Washington University in St. Louis, MO 63130 \\ ${ }^{4}$ Jet Propulsion Laboratory, Pasadena, CA 91109
}

\begin{abstract}
Galactic cosmic ray (GCR) secondary/primary ratios such as $\mathrm{B} / \mathrm{C}$ and $(\mathrm{Sc}+\mathrm{Ti}+\mathrm{V}) / \mathrm{Fe}$ are commonly used to determine the mean amount of interstellar material through which cosmic rays travel before escaping from the Galaxy $\left(\Lambda_{e s c}\right)$. These ratios are observed to be energy-dependent, with a relative maximum at $\sim 1 \mathrm{GeV} / \mathrm{nucleon}$, implying a corresponding peak in $\Lambda_{e s c}$. The decrease in $\Lambda_{e s c}$ at energies above $1 \mathrm{GeV} /$ nucleon is commonly taken to indicate that higher energy cosmic rays escape more easily from the Galaxy. The decrease in $\Lambda_{\text {esc }}$ at energies $<1 \mathrm{GeV} / \mathrm{nuc}$ is more controversial; suggested possibilities include the effects of a galactic wind or the effects of distributed acceleration of cosmic rays as they pass through the interstellar medium. We consider two possible explanations for the low energy decrease in $\Lambda_{e s c}$ and attempt to fit the combined, high-resolution measurements of secondary/primary ratios from $\sim 0.1$ to $35 \mathrm{GeV} /$ nuc made with the CRIS instrument on ACE and the $\mathrm{C} 2$ experiment on HEAO-3. The first possibility, which hypothesizes an additional, local component of low-energy cosmic rays that has passed through very little material, is found to have difficulty simultaneously accounting for the abundance of both $\mathrm{B}$ and the Fe-secondaries. The second possibility, suggested by Soutoul and Ptuskin, involves a new form for $\Lambda_{e s c}$ motivated by their diffusion-convection model of cosmic rays in the Galaxy. Their suggested form for $\Lambda_{e s c}(E)$ is found to provide an excellent fit to the combined ACE and HEAO data sets.
\end{abstract}

\section{INTRODUCTION}

The simplest form of the leaky box model of GCR propagation (1) is characterized by an exponential pathlength distribution (PLD) for the GCRs. The mean of this PLD is $\Lambda_{e s c}(E)$ (where $E$ denotes energy/nucleon). The energy dependence of $\Lambda_{e s c}(E)$ is a free parameter in the model, and GCR secondary/primary ratios and spectra can be used to deduce empirically the form of this energy dependence. At high energies, the form of $\Lambda_{e s c}(E)$ is constrained by the high-precision HEAO3-C2 data from a $\sim 1 \mathrm{GeV} /$ nucleon up to $\sim 35 \mathrm{GeV} /$ nucleon (2). Depending on the shape of the GCR source spectra input to the model, one finds $\Lambda_{e s c} \propto R^{\alpha}$, where $\alpha \simeq-0.6$ (see, eg. (3), (4)). This decrease in $\Lambda_{e s c}(E)$ at high energies is generally taken to indicate that GCRs escape more easily from the Galaxy at higher energies.

Below $\sim 1 \mathrm{GeV} /$ nucleon, the GCR secondary/primary ratios are observed to decrease, thus implying a decrease in $\Lambda_{e s c}(E)$ from a peak occurring at $\sim 1 \mathrm{GeV} /$ nucleon (see e.g. (3), (4)). Various theoretical explanations exist for a decrease in $\Lambda_{e s c}(E)$ towards lower energies, but none have gained widespread acceptance (see (5) and references therein). Many studies have simply introduced an artificial break in $\Lambda_{e s c}(E)$ at $1-2 \mathrm{GeV} /$ nucleon without theoretical justification. For example, a commonly used form is $\Lambda_{e s c}(E) \propto \beta^{\gamma}$, for $R<R_{0}$, where $R_{0}$ is a constant rigidity typically chosen to be $\sim 5 G V$, and $\gamma \sim 1$ to 4 .

The Cosmic Ray Isotope Spectrometer (CRIS) on NASA's Advanced Composition Explorer (ACE) spacecraft has made the most precise measurements to date of the solar minimum energy spectra of GCR nuclei with $\mathrm{Z}=4$ to 28 in the energy range $50-500 \mathrm{MeV} /$ nucleon. The data considered here (taken during the period August 1997 to April 1998) provide significantly tighter constraints on the form of $\Lambda_{\text {esc }}(E)$ at low energies than were previously possible. The data can also be used to test new interpretations of the observed low-energy decrease in secondary/primary ratios. In this paper, we attempt to avoid artificial fits to $\Lambda_{e s c}(E)$ and investigate whether introducing a second, low-energy source consisting of cosmic rays that have passed through very little matter (i.e. a relatively local source of cosmic rays) can reproduce the observations without invoking a decrease in $\Lambda_{e s c}(E)$

CP528, Acceleration and Transport of Energetic Particles Observed in the Heliosphere: ACE 2000 Symposium, edited by Richard A. Mewaldt, et al.

(C) 2000 American Institute of Physics 1-56396-951-3/00/\$17.00 
at low energies. We also consider a new form for $\Lambda_{e s c}(E)$ suggested by Soutoul and Ptuskin (6) that is motivated by a galactic diffusion-convection model of GCR propagation in the Galaxy.

\section{STANDARD LEAKY BOX MODEL}

We first attempt to fit the CRIS and HEAO3-C2 composition and energy spectra using a leaky box model with no local source of cosmic rays. We use a steady-state model based on the formalism of Meneguzzi, Audouze, and Reeves (1), described previously in (7), and (8). The model includes the effects of escape from the Galaxy, energy losses in and nuclear interactions with the ISM, and decay of radioactive species. The increased energy losses due to the ionized fraction of hydrogen in the ISM are accounted for as in (9), and the ISM is assumed to be $90 \%$ hydrogen and $10 \%$ helium by number. The source spectra for all GCRs are taken to be power laws in momentum, with indices of $-2.35\left(d Q / d E \propto P^{-2.35}\right)$, and the source elemental abundances used are within $5 \%$ of the source abundances quoted in the HEAO3-C2 analysis of Englemann et al. (2). Energy-dependent partial cross sections for the GCRs were calculated using the semi-empirical cross section formulae of Silberberg, Tsao and Barghouty (10), scaled to experimental data from (11) and (12) and references therein. Total inelastic cross sections were taken from (13) and references therein. Solar modulation was calculated using the spherically symmetric model of Fisk (14), with a solar wind speed of $400 \mathrm{~km} / \mathrm{s}$, a diffusion coefficient $\kappa(R)=\kappa_{0} \beta R$, where $R$ is the rigidity and $\beta$ the velocity of the cosmic ray, and a modulating volume extending to $120 \mathrm{AU}$.

We have investigated a variety of proposed forms for $\Lambda_{e s c}(E)$ but find that a parameterization proposed by Soutoul and Ptuskin (6) in the context of a diffusionconvection model of GCR propagation in the Galaxy provides the best fit to the data:

$$
\Lambda_{\mathrm{esc}}=\frac{29.5 \beta}{(\beta R)^{0.6}+(\beta R / 1.3 \mathrm{GV})^{-2.0}}
$$

Figures 1 and 2 show the results of the model fit to the $\mathrm{B} / \mathrm{C}$ and $(\mathrm{Sc}+\mathrm{Ti}+\mathrm{V}) / \mathrm{Fe}$ secondary/primary ratios, and the energy spectra. Also shown is the energy dependence of $\Lambda_{e s c}(E)$ used to obtain the fit. Equation 1 gives the usual $R^{-0.6}$ dependence at high energies. At low energies, $\Lambda_{e s c}(E) \propto \beta^{3} R^{2}$, a significantly stronger energydependence than used in many previous studies (e.g. (3) or (4)). This behavior at low energies is a consequence of the assumed presence of a galactic wind that convects cosmic rays from the Galaxy with a convection velocity which is a non-monotonic function of distance from the galactic plane. This form provides an excellent fit to the

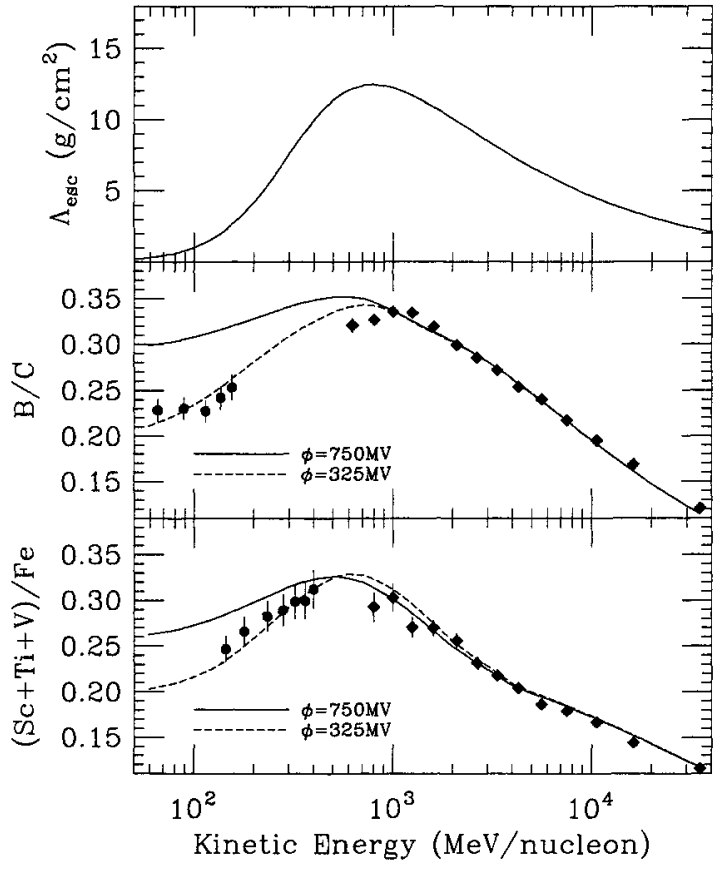

FIGURE 1. Leaky box model fits to CRIS (circles) and HEAO3-C2 (diamonds) $\mathrm{B} / \mathrm{C}$ and ( $\mathrm{Sc}+\mathrm{Ti}+\mathrm{V}$ )/Fe secondary/primary ratios. The top panel shows the energy dependence of $\Lambda_{e s c}(E)$ used to obtain the fit (Equation 1).

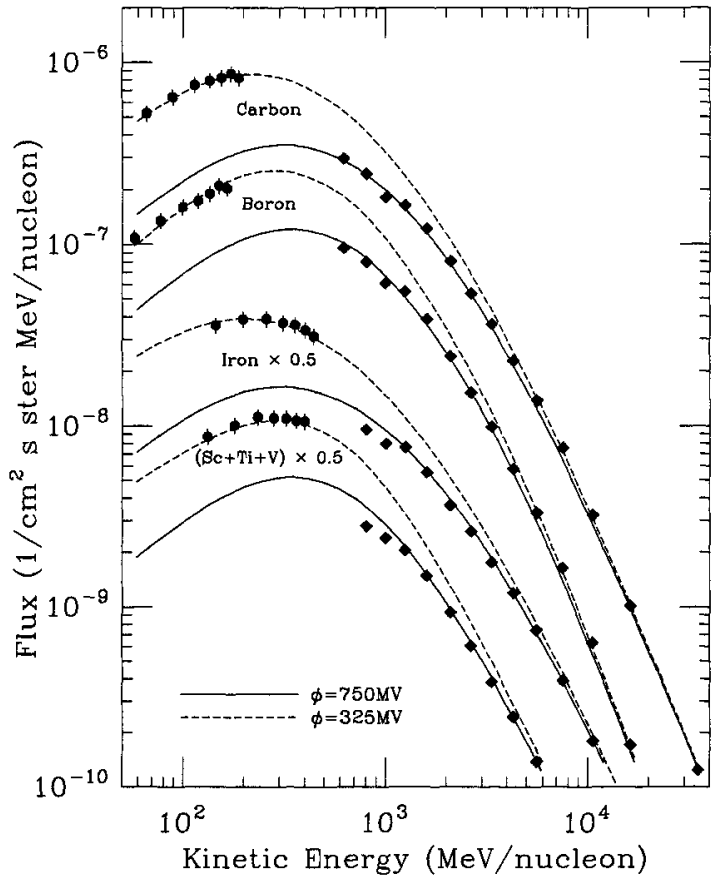

FIGURE 2. Leaky box model fits to CRIS (circles) and HEAO3-C2 (diamonds) B, C, Fe and ( $\mathrm{Sc}+\mathrm{Ti}+\mathrm{V}$ ) spectra, using $\Lambda_{e s c}(E)$ as in Equation 1.

new, high-precision CRIS data, and also data from previous space experiments, such as (7) and (15). Note that a 
strong energy-dependence of $\Lambda_{\text {esc }}(E)$ at low energies was also used in the analysis of (7).

The CRIS data were obtained during solar minimum, while the HEAO3-C2 data were obtained during intermediate solar modulation conditions in 1980 , so the two data sets cannot be fit using the same modulation parameters. Using our assumed source spectra, we find that a modulation parameter $\phi=325 \mathrm{MV}$ best fits the CRIS data, while $\phi=750 \mathrm{MV}$ best fits the HEAO3-C2 data.

\section{LEAKY BOX MODEL WITH A LOCAL SOURCE OF PRIMARY COSMIC RAYS}

We now consider a leaky box model without a strong decrease in $\Lambda_{\text {esc }}(E)$ at low energies: i.e.

$$
\Lambda_{\mathrm{esc}}=29.5 \beta R^{-0.6}, \text { for all } \mathrm{R}
$$

Figure 3 shows a plot of Equation 2. With this $\Lambda_{e s c}(E)$, the leaky box model over-produces secondary GCRs relative to primaries at low energies. However, by introducing a second, low-energy source of cosmic rays that have passed through very little matter, we may be able to again construct a model which fits the observations. This 'local source' of cosmic rays should have energy spectra which are steep relative to GCR interstellar spectra, so that they contribute significantly only below $\sim 1 \mathrm{GeV} /$ nucleon. The composition of this local source would be determined by requiring that the combination of local-source CRs plus GCRs fit the observations.

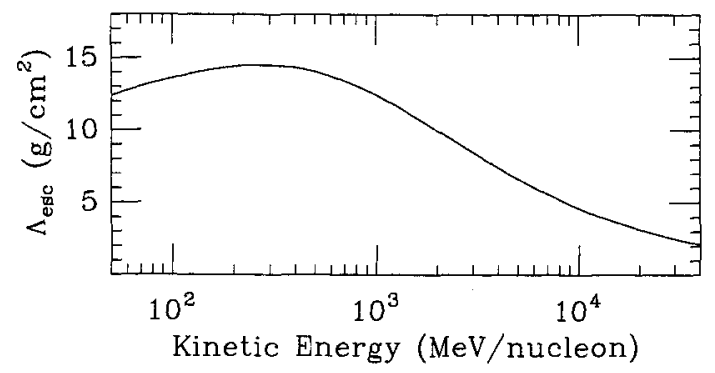

FIGURE 3. $\Lambda_{\mathrm{esc}}=29.5 \beta R^{-0.6}$, for all $\mathrm{R}$

Figure 4 illustrates the idea. A GCR interstellar carbon spectrum is shown, calculated using a leaky box model with $\Lambda_{e s c}(E)$ as in Equation 2. Also shown is a possible local-source carbon spectrum, with $Q(E) \propto$ $E^{-1} \exp \left(-E / E_{0}\right)$ where $E_{0}=500 \mathrm{MeV} /$ nucleon (the 'superbubble' spectrum of Bykov and Fleishman (16)). After modulating these spectra to $400 \mathrm{MV}$, the local source contributes a significant fraction of the total carbon intensity at $\sim 200 \mathrm{MeV} /$ nucleon, while contributing negligibly above $1 \mathrm{GeV} /$ nucleon.

Since CRs from the local source are assumed to travel through little or no material before reaching the solar sys-

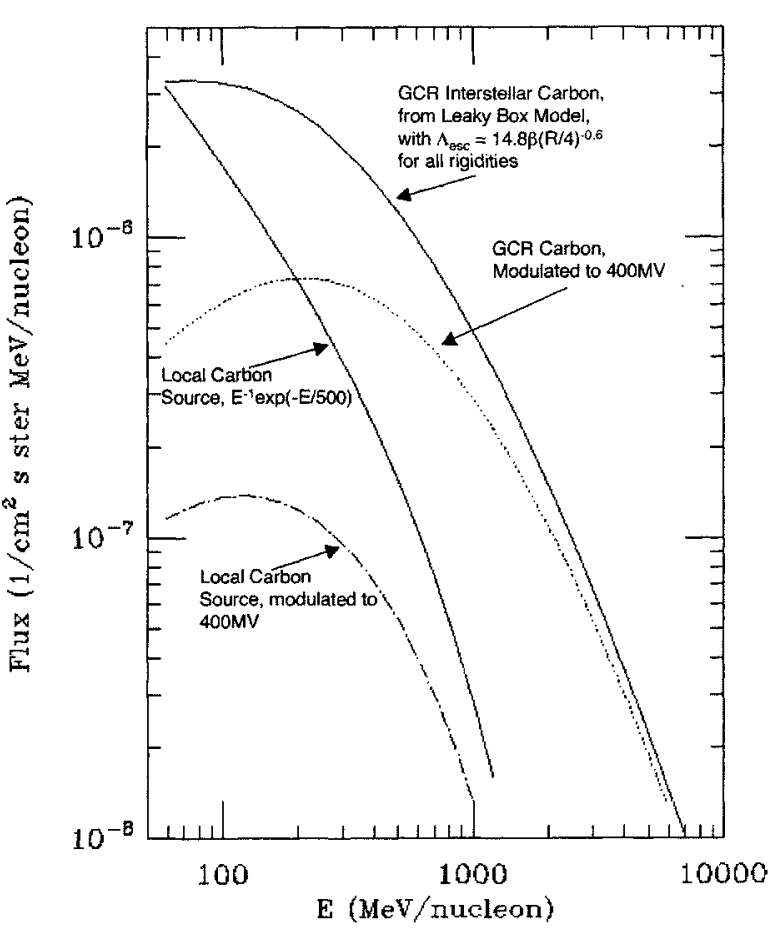

FIGURE 4. Schematic illustration of interstellar and modulated GCR and local source carbon spectra. The local source contributes significantly to the total carbon spectrum only below $\sim 1 \mathrm{GeV} /$ nucleon.

tem, they cannot contribute any secondaries to the mix. Therefore all the secondaries must be produced by the GCR component, and we must try to fit the CRIS and HEAO boron and $(\mathrm{Sc}+\mathrm{Ti}+\mathrm{V})$ spectra with the leaky box model using $\Lambda_{e s c}(E)$ as in Equation 2. The spectra of primary elements such as $\mathrm{C}$ and $\mathrm{Fe}$ are taken to be an appropriate mix of the GCR and local components.

Figures 5 and 6 show our attempt to achieve this fit. In Figure 5 the modulation level for the CRIS data is adjusted to $475 \mathrm{MV}$, which fits the CRIS boron spectrum. In Figure 6 a local source of $\mathrm{C}$ and $\mathrm{Fe}$ have been added in, which makes it possible to fit the measured CRIS and HEAO spectra for these elements. The required local contributions of $\mathrm{C}$ and $\mathrm{Fe}$ (evaluated at $200 \mathrm{MeV} /$ nuc at $1 \mathrm{AU}$ with $\phi=475 \mathrm{MV}$ ) amount to $22 \%$ and $35 \%$ of the GCR $\mathrm{C}$ and $\mathrm{Fe}$ at this energy. While the resulting fits to $\mathrm{B}, \mathrm{C}$, and Fe are all satisfactory at both CRIS and HEAO modulation levels, note in Figure 5 that this model does not produce sufficient $\mathrm{Fe}$-secondaries. Indeed, using this form for $\Lambda_{e s c}(E)$ we were unable to find any combination of parameters that provided satisfactory fits to both the boron and Fe-secondaries at the same level of solar modulation. While this does not rule out the possibility of contributions from a local source, it does indicate that our particular choice of $\Lambda_{e s c}(E)$ and local source spectra do not lead to an improved fit to the available GCR data. 


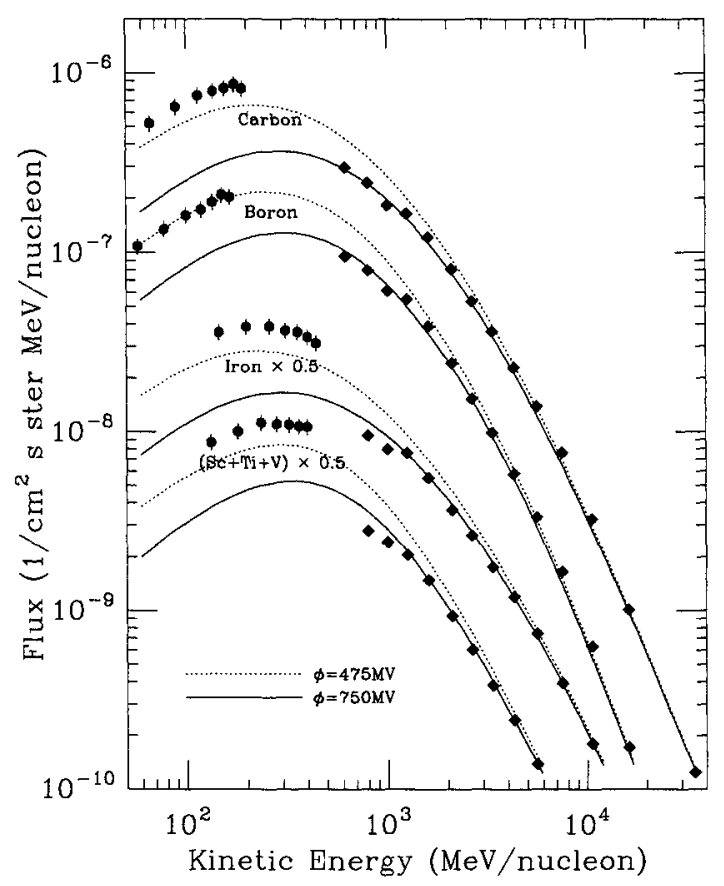

FIGURE 5. Comparison of leaky box model results using $\Lambda_{\text {esc }}(E)$ as in Equation 2 with CRIS and HEAO3-C2 B, C, Fe and $(\mathrm{Sc}+\mathrm{T} \mathrm{T}+\mathrm{V})$ spectra. The boron and sub-Fe secondaries cannot be fit simultaneously.

\section{CONCLUSIONS}

The leaky box model of GCR propagation successfully reproduces the combined ACE and HEAO observations. However, this fit requires a stronger energy-dependence of $\Lambda_{\text {esc }}(E)$ at energies below $\sim 1 \mathrm{GeV} /$ nucleon than has been used in many previous studies. We find that the functional form proposed by Soutoul and Ptuskin (6) in the context of a galactic diffusion-convection model provides an excellent fit to the data.

We investigated whether introducing a second, lowenergy, local source of cosmic rays might lead to a model which also reproduces the observations, without invoking a decrease in $\Lambda_{e s c}(E)$ at low energies. This approach does not lead to an improved fit to the data, since it does not reproduce the GCR secondaries as well as the standard leaky box model. However, a local source of cosmic rays in combination with other forms of $\Lambda_{e s c}(E)$ is not ruled out. CRIS data spanning a complete solar cycle will allow for more precise tests of this idea during the coming years, since the relative contributions of the local and GCR components arriving at $1 \mathrm{AU}$ should change as a function of the level of solar modulation.

\section{ACKNOWLEDGEMENTS}

This research supported by NASA at Caltech (grant NAG5-6912), JPL, NASA/GSFC, and Washington U.

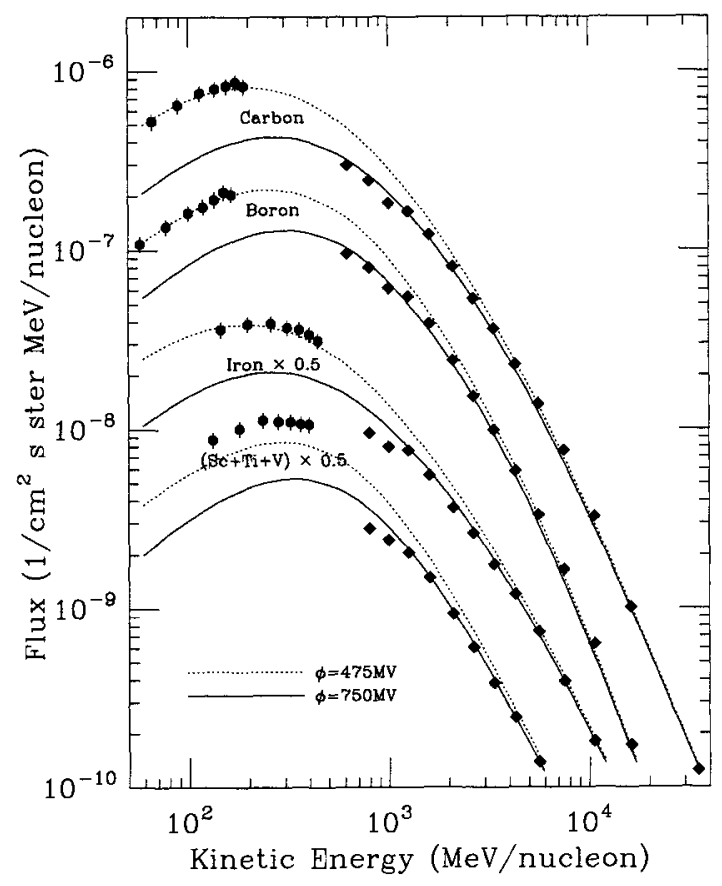

FIGURE 6. As in Figure 5, with $\mathrm{C}$ and Fe from the proposed local source added to the GCRs from the leaky box model.

\section{REFERENCES}

1. Meneguzzi, M., Audouze, J., and Reeves, H., Astron. Astrophys. 15, 337 (1971).

2. Englemann, et al., Astron. Astrophys. 233, 96-111 (1990).

3. Stephens, S. A., and Streitmatter, R., Astrophys. J. 505, 266-277 (1998).

4. Webber, W. R., et al., Astrophys. J. 457, 435-439 (1996).

5. Ptuskin, V. S., This Volume.

6. Soutoul, A., and Ptuskin, V. S., Proc. 26th Int. Cosmic Ray Conf. (Salt Lake City) 4, 184-186 (1999).

7. Krombel, K. E., and Wiedenbeck, M. E., Astrophys. J. 328, 940-953 (1988).

8. Leske, R. A., Astrophys. J. 405, 567-583 (1993).

9. Soutoul, A., Ferrando, P., and Webber, W. R., Proc. 21st Int. Cosmic Ray Conf. (Adelaide) 3, 337-341 (1990).

10. Silberberg, R., Tsao, C. H., and Barghouty, A. F., Astrophys. J. 501, 911-919 (1998).

11. Webber, W. R., et al., Astrophys. J. 508, 940-948 (1998).

12. Webber, W. R., et al., Astrophys. J. 508, 949-958 (1998).

13. Tripathi, R. K., Cucinotta, F. A., and Wilson, J. W., NASA Technical Paper 3621 (1997).

14. Fisk, L. A., J. Geophys. Res. 76, 221 (1971).

15. Garcia-Munoz, M., and Simpson, J. A., Proc. 16th Int. Cosmic Ray Conf. (Kyoto) 1, 270-274 (1979).

16. Bykov, A. M., and Fleishman, G. D., MNRAS 255, 269 (1992). 